\title{
An Overview of Signal Processing for Food Inspection
}

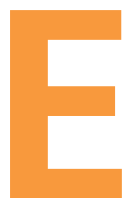

very year, millions of people experience serious-and sometimes fatal-health problems following consumption of unsafe or contaminated food. The contamination may involve foodborne disease or chemical hazards. Furthermore, billions of dollars are lost annually in the food industry to insect damage and inefficient production and inspection processes. The goal of this article is to introduce the signal processing community to the challenges that arise in food inspection. We briefly describe both traditional food-inspection technologies, which rely on sample collection and subsequent offline analysis in a laboratory, and newer approaches that use nondestructive methods to measure various quality parameters of food products in real time. We focus on four specific examples to illustrate the breadth of technologies currently in use in food inspection and the challenges that remain to be addressed. In each case, we describe the problem setting and its economic and health aspects; the techniques that are used, including the physical principles on which these techniques are based; and their performance and cost.

\section{PISTACHIO SORTING}

Food inspection in pistachio sorting involves identifying the closed-shell pistachio nuts. Although the characteristic split in pistachio nuts occurs on the tree well before harvest, approximately $20 \%$ of the harvest contains nuts with closed shells. These nuts are of much lower value than the open-shell nuts, which are sold as snack foods. In some countries, separation of open- and closed-shell nuts is mostly performed by manual labor, which poses an obvious sanitary problem. However, in many countries, closed- shell pistachio nuts are removed by mechanical devices that are known as "pinpickers." A pinpicker is a large drum lying on its side, the inside of which is lined with needles. Pistachios are placed into a rotating pinpicker where the needles slip into the open shell and lift them away while closed-shell nuts fall out the end. These devices have low classification accuracy and can damage kernels in open-shell pistachios by pricking them with the needle. Also, the needle hole can give the appearance of an insect tunnel and cause rejection by the consumer.

To address these problems, acoustics (already used in agriculture to measure fruit firmness and watermelon quality) has been considered for rapid monitoring of tree nut qualities [1], [2]. An impact-acoustics-based sorting system has been developed to separate pistachio nuts with closed shells from those with open shells. The sorting system, shown in Figure 1, consists of a microphone, digital signal processing hardware, material-handling equipment, and an air reject mechanism. Upon impact with a steel plate, nuts with closed shells emit different sounds than nuts with open shells and can be rejected. In the sorting system, mel-cepstrum and principal component analysis (PCA) procedures are used to produce features extracted from the microphone signal during the first $1.4 \mathrm{~ms}$ after impact [3]. A classification accuracy of $99 \%$ is achieved for the closed-shell nuts on the test set, which did not include the training set. An important property of the algorithm is that it is easily trainable, as are many speech-recognition algorithms. This acoustic sorting system is noninvasive, highly adaptable, and provides $10 \%$ higher sorting accuracy than mechanical sorting systems with lower-cost hardware.

\section{SIGNAL PROCESSING CHALLENGES}

Noise from adjacent machines can somewhat reduce the classification accuracy. Thus, there is a need for noise canceling algorithms to improve accuracy in noisy environments, such as food processing facilities. A flexible training scheme will be a very important feature for a plant because pistachios differ from region to region, and training the machine just before classifying a new load increases the classification accuracy.

\section{WHEAT INSPECTION}

Food inspection in wheat involves the identification of internal insect infestation of wheat kernels, which degrades quality and value of wheat and is one of the most difficult defects to detect. Wheat kernels become infested when an adult female insect chews a small hole, (about $0.05 \mathrm{~mm}$ in diameter), into the kernel, deposits an egg, and then seals the egg with a mixture of mucus and wheat that was chewed out. The egg plug is the same color as the wheat surface, so it is nearly impossible to detect by external examination. When the egg hatches, the insect larvae develops and consumes tunnels inside the wheat kernel until it reaches maturity. Finally, the insect exits the kernel by chewing an exit tunnel. Wheat millers usually specify that wheat loads must contain fewer than five insect-damaged kernels (IDK) per $100 \mathrm{~g}$ (approximately 3,000 kernels). Inspecting for IDKs is labor intensive and may miss most of the infested kernels where an immature insect has not emerged from the kernel.

Several methods have been used, or are currently under development, to detect insect damage inside whole wheat kernels. Previously developed methods for IDK detection fall into two categories: 
bulk sample analysis and single kernel inspection. While bulk sample analysis is less labor intensive and usually less cost$l y$, it cannot detect insect infestations at low levels such as five or ten kernels per $100 \mathrm{~g}$. The only method to detect IDK at these low levels is by inspecting single kernels very rapidly. Bulk methods that have been developed include flotation methods, acoustic detection of larval movement and chewing [5], carbon dioxide measurements, and staining of amino acids specific to insect body fluids. Single kernel methods include x-ray imaging [6], near-infrared spectroscopy [7], signal processing of forces exerted on the kernel as it is being crushed and, more recently, impact-acoustic techniques where the acoustic signal generated when kernels impact a metallic plate is processed [4]. Of all of the single kernel methods, x-ray imaging is the most accurate and is not greatly affected by different types of wheat while all the other methods are affected. However, for x-ray imaging, good image segmentation methods have not yet been developed to isolate wheat kernels that may be touching or overlapping. X-ray imaging is also costly, with the equipment costing US $\$ 100,000$ or more.

\section{SIGNAL PROCESSING CHALLENGES}

The impact-acoustic method can inspect wheat at high rates, as with the acoustical pistachio sorting method. However, more research is needed to improve its accuracy. The current classification rate is about $80 \%$ [4]. This has to reach above $90 \%$ for a practical system.

\section{MYCOTOXIN DETECTION}

Food inspection for mycotoxin detection involves screening agricultural products to identify a class of powerful carcinogens produced by natural molds prevalent throughout the world. The two most common mycotoxins are aflatoxin and fumonisin, produced by the molds Aspergillus flavus and Fusarium verticillioides, respectively. Mycotoxins are found in many raw agricultural products, especially corn, peanuts, tree nuts, cotton seed, and figs. To reduce the risks to animal and human health, the United States Food and Drug Administration
(FDA) requires that foods intended for consumption and for use in feeding of dairy animals, and of immature animals contain less than 20 parts per billion (ppb) aflatoxin and 2.0 parts per million (ppm) fumonisin. Regulations in Europe are more stringent. Incidence of mycotoxin contamination increases if grain is not properly dried and stored after harvest, and with drought stress; thus, almost every year, some isolated farming locations produce corn containing mycotoxins exceeding FDA limits.

For corn loads contaminated with mycotoxins beyond FDA limits, only a very small percentage (less than 1\%) of individual corn kernels actually contains mycotoxins. However, these are contaminated at such high levels that they can cause a large sample to contain, on average, levels of mycotoxins beyond regulated levels. As such, the food industry is in need of methods to detect and remove kernels that are contaminated by mycotoxins. If the small percentage of contaminated kernels is separated, then corn-based food products will become safer, with a minimal loss of product due to sorting. Currently, loads containing moderate amounts of aflatoxin are blended with uncontaminated corn to lower the concentration of aflatoxin. This grain is then fed to livestock. Loads with high amounts of aflatoxin must be destroyed, costing the industry millions of dollars in some years.
There are high-speed optical sorters available that can sort corn at rates up to $10,000 \mathrm{~kg} / \mathrm{h}$; examples include those produced by Satake USA Inc., Sortex, and Key Technology. These machines sort by measuring light reflectance at two or three spectral bands in the visible or near-infrared region (NIR) of the spectrum, between 400 and 1,700 nm. To utilize these sorters for a given application, one must determine which two or three spectral bands are optimal for discriminating the product that is to be accepted and rejected.

\section{SIGNAL PROCESSING CHALLENGES}

Instead of arbitrarily selecting two spectral bands, every combination of two spectral bands in the spectra for optimal separation of aflatoxin-contaminated kernels can be searched exhaustively [8]. This method was tested in a high-speed sorter for its performance in reducing aflatoxin and fumonisin and was shown to reduce both mycotoxin levels by $85-90 \%$, while removing only $5 \%$ of the incoming product. Another approach is to design a sorting device based on the decay rate of the NIR spectrum around $750 \mathrm{~nm}$, as shown in Figure 2. The NIR spectra of good kernels have a much sharper decay around $750 \mathrm{~nm}$ compared to contaminated kernels. Similar procedures have been applied to separating defects in pistachio nuts, and this

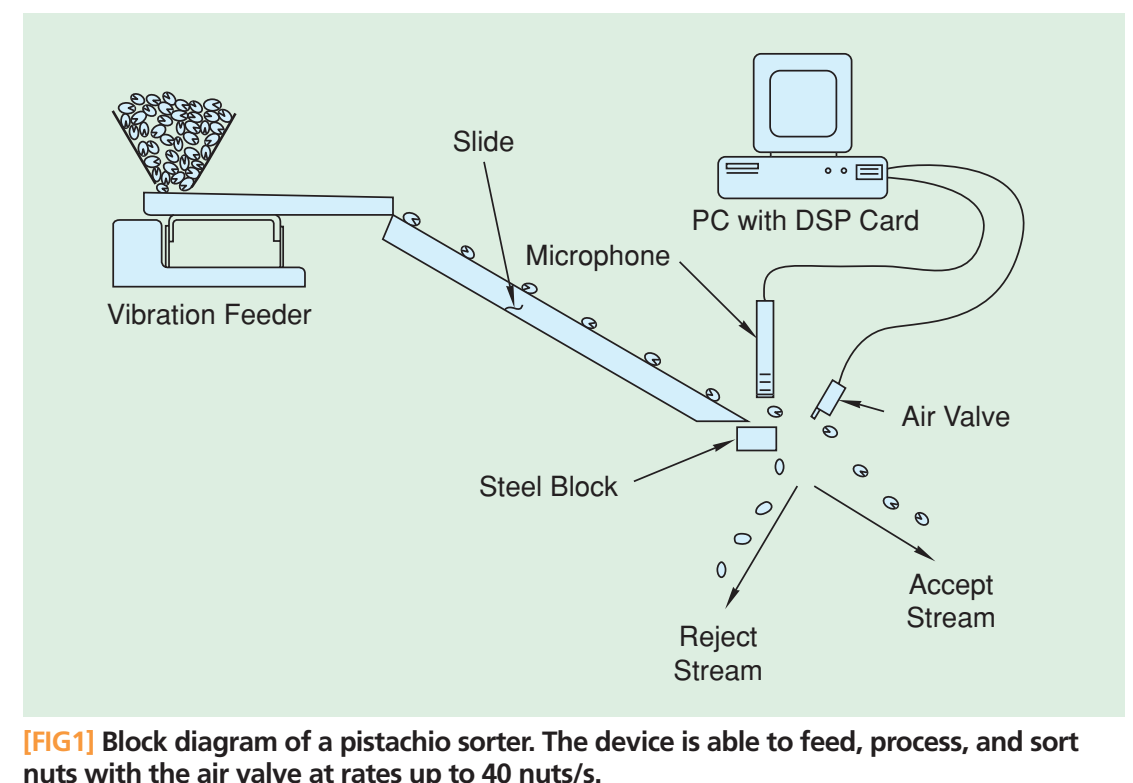
[FIG 1] Block diagram of a pistachio sorter. The device is able to feed, process, and sort
nuts with the air valve at rates up to 40 nuts/s. 


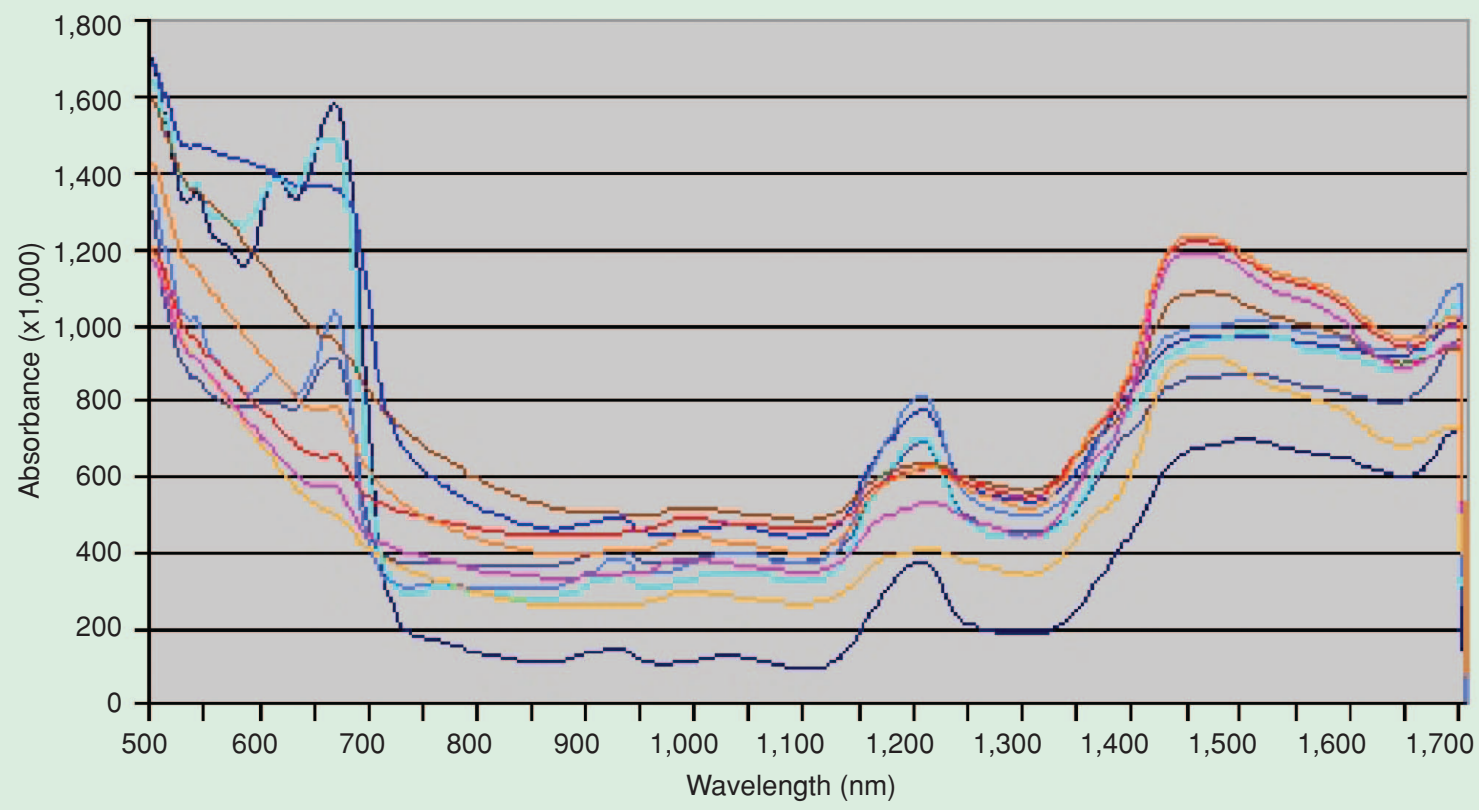

[FIG2] Near-infrared region of the spectrum of good corn kernels (in blue) and contaminated corn kernels (in red). The decay rate around $750 \mathrm{~nm}$ can be used to classify the kernels.

method is also being studied for its efficacy in removing aflatoxin-contaminated hazelnuts and mold-damaged wheat [9].

\section{ACRYLAMIDE DETECTION}

Food inspection for acrylamide detection involves the detection of this wellknown neurotoxin in French fries and fried potato chips using image processing. Acrylamide is classified as a probable human carcinogen by the International Agency for Research on Cancer (IARC). In 2002, Swedish researchers [10] found that potato chips and French fries contain levels of acrylamide that are hundreds of times higher than those considered safe for drinking water by the Environmental Protection Agency (EPA) and the World Health Organization (WHO). Currently, chemical methods are used to estimate acrylamide levels in baked or fried foods. These methods usually entail extraction of acrylamide from food and purification of the extract prior to analysis by liquid chromatography or gas chromatography coupled with mass spectrometry. The associated analytical systems are very expensive and not common in food inspection laboratories.
On the other hand, chemical reactions on the surface of foods are responsible for the formation of color and acrylamide, giving them an opportunity to correlate with each other. A simple color-measurement device measuring CIE Lab parameters cannot be used to estimate meaningful parameters for acrylamide levels in a given food item because the color is not homogenously distributed over the surface of the food item.

Fortunately, the image of a food item can be analyzed in real time, and meaningful features correlated with the acrylamide level can be estimated from the image of the food item. After the frying process, bright yellow, brownish yellow, and dark brown regions are clearly visible in potato images. We experimentally observed a high correlation between the normalized acrylamide level and the normalized ratio of brownish yellow regions to the total area in a fried potato chip. Specifically, we can segment a given potato image into three regions using an image segmentation algorithm. The higher the number of brownish yellow pixels, the higher the acrylamide level in a fried potato chip. This observation indi- cates that, by installing cameras in packaging lines, and analyzing potato chip images in real time, one can detect and remove potato chips with brownish yellow regions from a production line, significantly reducing the acrylamide levels that people consume.

\section{SIGNAL PROCESSING CHALLENGES}

Unsupervised image segmentation with automated feature selection capability is needed to solve future image-based food inspection problems. Food items can also be imaged using UV and IR cameras or even X-ray imaging systems. Typically, a plant manager simply wants to train the signal and image processing system with faulty items and regular items and would like the system to figure out the classification by itself.

\section{CONCLUSIONS}

There are several applications in agriculture waiting for innovative signal processing methods [1]-[9]. Since applications in agriculture must be performed quickly and at low cost, there is a need to develop simple and effective methods for extracting useful

(continued on page 122) 


\section{AUTHORS}

Sergios Theodoridis (stheodor@di. uoa.gr) is with the University of Athens, Greece. His research interests lie in the areas of signal processing and pattern recognition. He is the coauthor of the book Pattern Recognition, Academic Press, 2006, currently in its third edition. $\mathrm{He}$ is the President of the European Association for Signal Processing (EURASIP), a fellow of IEE, and a Senior Member of the IEEE.

Michael Mavroforakis (mmavrof@di. uoa.gr) is with the University of Athens,
Greece. His research interests lie in the areas of pattern recognition and medical imaging. He is the recipient of the EUSIPCO-2005 best student paper award for an article coauthored with $\mathrm{S}$. Theodoridis.

\section{REFERENCES}

[1] V.N. Vapnik, Statistical Learning Theory. New York: Wiley, 1998.

[2] S. Theodoridis and K. Koutroumbas, Pattern Recognition, 3rd ed. New York: Academic, 2006.

[3] D.J. Crisp and C.J.C. Burges, "A geometric interpretation of $\mathcal{V}$-SVM classifier," in Proc. Neural Information Processing, 2000, vol. 12, pp. 244-250.

[4] B. Schölkopf, A.J. Smola, R.C. Williamson, and P.L. Bartlett, "New support vector algorithms,"
Neural Comput., vol. 12, no. 5, pp. 1207-1245, 2000.

[5] E.G. Gilbert, "An iterative procedure for computing the minimum of a quadratic form on a convex set," SIAM J. Control, vol. 4, no. 1, pp. 61-79, 1966.

[6] M. Mavroforakis and S. Theodoridis, "A geometric approach to support vector machine classification," IEEE Trans. Neural Networks, vol. 17, no. 3, pp. 671-682, 2006.

[7] M. Mavroforakis, M. Sdralis, and S. Theodoridis, "A novel SVM algorithm based on reduced convex hulls," in Proc. Int. Conf. Pattern Recognition (ICPR), 2006, pp. 564-568.

[8] Q. Tao, G.-W. Wu, and J. Wang, "A generalized S-K algorithm for learning $v$-SVM classifiers," Pattern Recognit. Lett., vol. 25, no. 10, pp. 1165-1171, 2004.

[9] S.S. Keerthi, S.K. Shevade, C. Bhattacharyya, and K.R.K. Murthy, "Improvements to Platt's SMO algorithm for SVM classifier design," Neural Comput., vol. 13, no. 3, pp. 637-649, 2001.

applications CORNER continued from page 108

and effective methods for extracting useful features from signals and images captured from food samples and for classification of large sets of features. In particular, it is expected that food inspection will require more automated equipment to perform these tasks, for which machine learning and classification algorithms with automated feature selection are good candidates. Therefore, there are many opportunities for the signal processing community to contribute to food inspection and other agriculture applications.

\section{ACKNOWLEDGMENT}

This work was funded in part by the National Science Foundation (NSF) and the Turkish Scientific and Technical Research Council (TUBITAK).

\section{AUTHORS}

Tom Pearson (thomas.pearson@gmprc. ksu.edu) is with the United States Department of Agriculture, Agricultural Research Service (USDA-ARS).

A. Enis Cetin (cetin@bilkent.edu.tr) is with Department of Electrical and Electronics Engineering, Bilkent University, Turkey.

Ahmed H.Tewfik (tewfik@umn.edu) is with the Department of Electrical and Computer Engineering, the University of Minnesota, United States.

Vural Gökmen (vgokmen@hacettepe. edu.tr) is with Food Engineering Department, Hacettepe University, Turkey.

\section{REFERENCES}

[1] J. Sugiyama, T. Katsurai, J. Hong, H. Koyama, and K. Mikuriya., "Melon ripeness monitoring by a portable firmness tester," Trans. ASAE, vol. 41, no. 1, pp. 121-127, 1998.

[2] M.L. Stone, P.R. Armstrong, D.D. Chen, G.H. Brusewitz, and N.O. Maness, "Peach firmness prediction by multiple location impulse testing," Trans. ASAE, vol. 41, no. 1, pp. 115-119, 1998.

[3] A.E. Cetin, T.C. Pearson, and A.H. Tewfik, "Classification of closed- and open-shell pistachio nuts using voice-recognition technology," Trans. $A S A E$, vol. 47, no. 2, pp. 659-664, 2004.

[4] T.C. Pearson, A.E. Cetin, and A.H. Tewfik, "Detection of insect damaged wheat kernels by impact-acoustic emissions," in Proc. 2005 IEEE Int. Conf. Acoustics, Speech, and Signal Processing (ICASSP 2005).

[5] R.W. Mankin, D. Shuman, and J.A. Coffelt, "Acoustic counting of adult insects with differing rates and intensities of sound production in stored wheat," J. Econ. Entom., vol. 90, no. 4, pp. 1032-1038, 1997.

[6] R.P. Haff, "Real-time x-ray inspection of wheat for infestation by the granary weevil, Sitophilus Granarius (L.)," Trans. ASAE, vol. 47, no. 2, pp. 531-537, 2004.

[7] E.B. Maghirang, F.E. Dowell, J.E. Baker, and J.E. Throne, "Automated detection of single wheat kernels containing live or dead insects using nearinfrared reflectance spectroscopy," Trans. ASAE, vol. 46, no. 4, pp. 1277-1282, 2003.

[8] T.C. Pearson, D.T. Wicklow, and M.C. Pasikatan, "Reduction of Aflatoxin and Fumonisin contamination in yellow corn by high-speed dual-wavelength sorting," Cereal Chem., vol. 81, no. 4, pp. 490-498, 2004.

[9] S. Delwiche, "Classification of scab- and other mold-damaged wheat kernels by near-infrared reflectance spectroscopy," Trans. ASAE, vol. 46, no. 3, pp. 731-738, 2003.

[10] K. Svensson, L. Abramsson, W. Becker, A. Glynn, K.E. Hellenas, Y. Lind, and J. Rosen, "Dietary intake of acrylamide in Sweden," Food Chem. Toxicol., vol. 41, no. 11, pp. 1581-1586, 2003.

\section{SP}

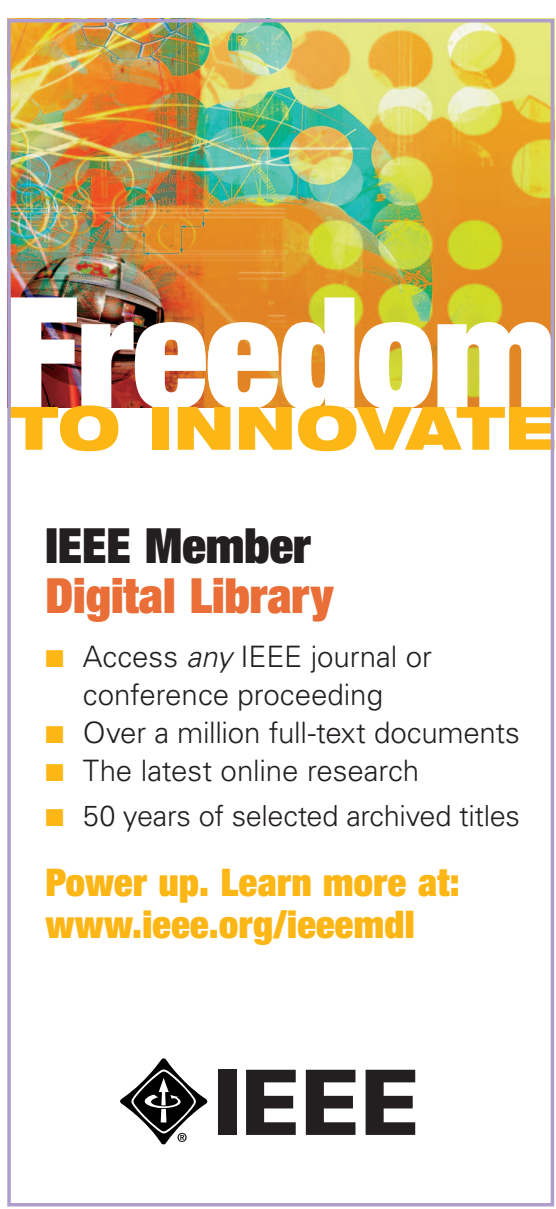

